\title{
An unusual abdominal mass
}

\author{
Talib Al-Mishlab, Tena Walters
}

A 78-year old woman presented with pain in her left lower abdomen for three days. She had also noticed an associated swelling in the same area, and a feeling of incomplete evacuation of her bowel. However, there was no nausea, vomiting, change in bowel habits, or rectal bleeding. She was otherwise fit and healthy, apart from mild hypertension for which she was taking bendrofluazide. On examination, she was anaemic and pyrexial. Pulse rate was 90 beats $/ \mathrm{min}$ and blood pressure $160 / 85 \mathrm{mmHg}$. Her abdomen was distended, with no scars, and normal hernial orifices. A tender, smooth, fixed, poorly outlined mass was found in the left iliac fossa. Rectal examination and sigmoidoscopy up to $12 \mathrm{~cm}$ were normal.

Initial investigations showed haemoglobin $9.8 \mathrm{~g} / 1$, white blood cells $10.1 \times 10^{9} / 1$, platelets $328 \times$ $10^{9} / 1$, and a normal chest X-ray. Her plain abdominal X-ray is shown in figure 1 . An initial diagnosis of diverticular abscess was made, and she was started on antibiotics, clear fluids by mouth and analgesia. Subsequently, she underwent a computed tomography (CT) scan of the pelvis which is shown in figure 2.

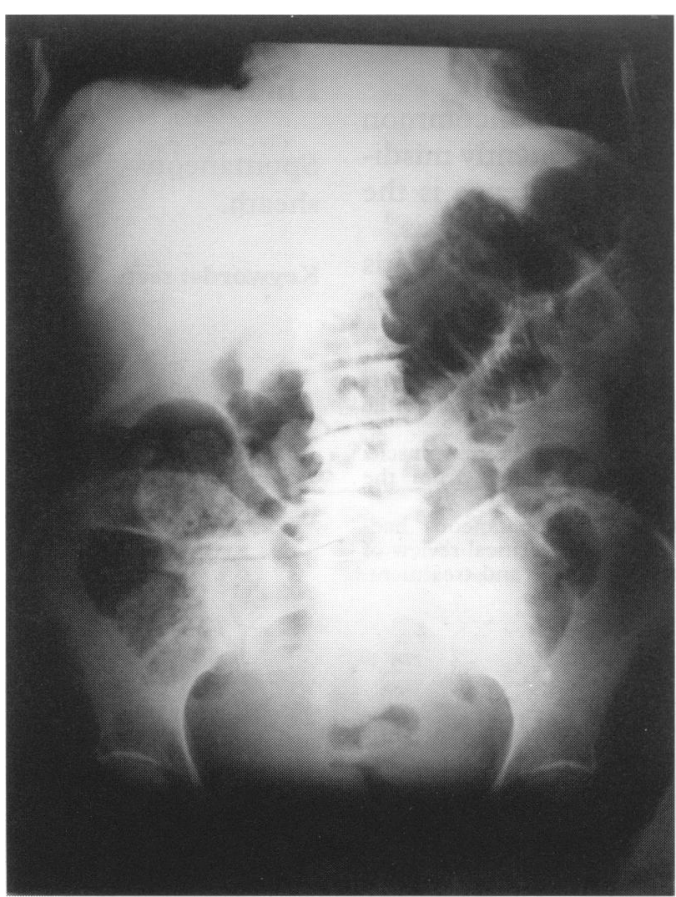

Figure 1 Plain abdominal X-ray

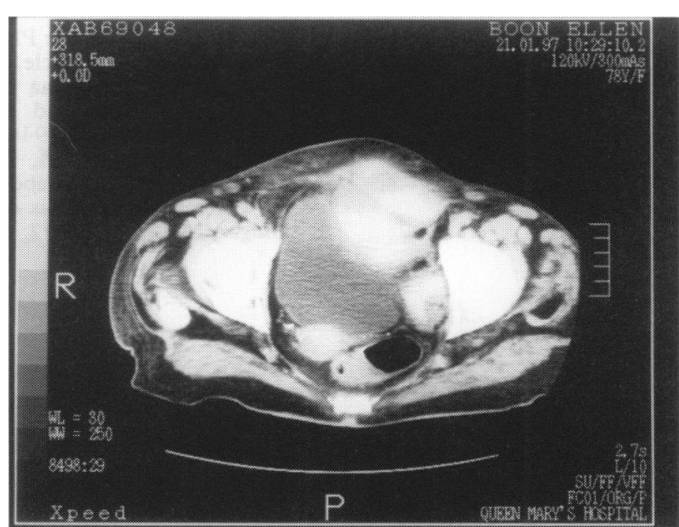

Figure 2 CT scan of pelvis
Surgery, Queen Mary's Hospital, Sidcup, Kent DA14 6LT, UK

T Al-Mishlab

T Walters

Correspondence to $\mathrm{Mr}$ Al-Mishlab, 9 Stane Way, Shooters Hill, London SE18 4PA, UK

Accepted 20 August 1997

\section{Questions}

1 What is shown on the plain abdominal X-ray?

2 What does the CT scan show?

3 Is laparotomy indicated? 


\section{Answers}

QUESTION 1

The plain abdominal X-ray (figure 1) shows dilated loops of small bowels with a soft tissue mass in the left iliac fossa consistent with bowel obstruction, probably caused by the soft tissue mass.

QUESTION 2

There is a large, haemorrhagic lesion, involving the lower part of the left rectus muscle and extends inferiorly in the left side of the pelvis. The lesion displaces the bladder and adjacent loops of bowel to the right but there is no evidence of invasion. The CT appearances are in keeping with a haematoma of the left rectus muscle (figure 2).

\section{QUESTION 3}

Laparotomy is not indicated. Conservative treatment with repeat scanning to monitor the resolution of the haematoma is the treatment of choice. However, such treatment can sometimes be futile and simple surgical evacuation can prove effective.

\section{Discussion}

Rectus sheath haematoma is an uncommon cause of abdominal pain. It is frequently misdiagnosed, and awareness of its existence is the single most important factor in its diagnosis. ${ }^{1}$

The aetiology of rectus sheath haematoma is spontaneous rupture of the superior or inferior

1 Verhagen HJ, Tolenaar PL, Sybrandy R. Haematoma of the rectus abdominis muscle. Eur $₹$ Surg 1993;59:335-8.

2 Teske JM. Haematoma of the rectus abdominis muscle: report of a case amd analysis of 100 cases from the literature. Am $\mathcal{F}$ Surg 1946;71:689-95.

3 Titone C, Lipsius M, Krakauer JS. Spontaneous haematoma of the rectus abdominis muscle. Critical review of 50 cases with emphasis on early diagnosis and treatment. Surgery 1972;72:568-72. epigastric arteries within the rectus abdominis muscle. This is commonly precipitated by coughing, and/or systemic anticoagulation therapy. It can also be caused by trauma, pregnancy, exertion and hypertension. It presents with pain and swelling in one of the four quadrants of the abdomen, commonly the lower quadrants, with the left upper quadrant being the rarest site.

Differential diagnosis includes acute appendicitis, strangulated hernia, twisted ovarian cyst, intestinal obstruction, tumours, or colonic perforation. ${ }^{3}$ The diagnosis can be confirmed by ultrasound, CT scan, or magnetic resonance imaging (MRI). CT scan is more diagnostic than ultrasound scan, but MRI is very useful if the CT scan findings are not specific for rectus sheath haematoma. ${ }^{4}$

Once the correct diagnosis has been made, management is usually conservative, consisting of analgesia, correction of the underlying coagulation defect, blood transfusion, and imaging monitoring of the resolution of the haematoma. Surgery is indicated for severe pain, and consists of evacuation of the clot and control of the bleeding. ${ }^{5}$

\section{Final diagnosis}

Spontaneous haematoma of the left rectus sheath.

Keywords: rectus sheath haematoma; abdominal mass

4 Fukuda T, Sakamoto J, Kohzaki S, et al. Spontaneous rectus sheath haematoma: clinical and radiological features. Abdom Imag 1996;21:58-61.

5 Way LW. Current surgical diagnosis and treatment, 9th edn. California: Appleton and Lange, 1990; pp 711. 